\title{
Análisis del comercio en Colombia: Unión Europea desde el uso de los indicadores de comercio exterior
}

\author{
Volumen XIX No (1). Enero-Junio 2019. Pág. 11-24 \\ ISSN: 0121-1048 IMPRESO ISSN: 2422-3220 EN LÍNEA \\ Torres Franco, Michael* \\ Universidad EAN, Facultad de Administración, Finanzas y Ciencias \\ Económicas. Calle 79 No 11-10. Bogotá, Colombia. \\ metorres@universidadean.edu.co
}

\begin{abstract}
Resumen
La integración comercial ha permitido incrementar la interdependencia económica, social y cultural de las naciones; permitiendo con esto ofrecer más y mejores posibilidades de consumo, así como incrementar la oferta de producción. Los efectos son variados y dependen de las economías que negocian, así como de los bienes y servicios involucrados. Este artículo analiza el comercio exterior bilateral a partir de indicadores de posición comercial, fórmulas que toman datos de exportaciones e importaciones para determinar el grado de dependencia que tiene un país frente a otro mercado, mostrando en este caso que Colombia tiene una alta dependencia hacia el mercado europeo y en determinados productos primarios; dejando como conclusión y necesidad apremiante la diversificación de la oferta exportable para minimizar riesgos futuros.
\end{abstract}

Palabras clave: integración económica, comercio bilateral, indicadores, exportaciones, importaciones

Códigos JEL: C02, F14, F15, F53

\section{Colombia Trade Analysis: European Union trade from the use of foreign trade indicators}

\begin{abstract}
Commercial integration has allowed to increase economic, social and cultural interdependence of nations; allowing this to offer more and better consumption possibilities, as well as increase the supply of production. The effects are varied and depend on the economies that negotiate, as well as on the goods and services involved. This article analyzes bilateral foreign trade based on indicators of commercial position, formulas that take data from exports and imports to determine the degree of dependence that a country has with another market, showing in this case that Colombia has a high dependence on European market and in certain primary products; leaving as conclusion and pressing need the diversification of the exportable offer to minimize future risks.
\end{abstract}

Keywords: economic integration, bilateral trade, indicators, export, import

JEL Codes: C02, F14, F15, F53

• Citar: Torres Franco, Michael (2019). Análisis del comercio en Colombia: Unión Europea desde el uso de los indicadores de comercio exterior. Inquietud Empresarial, XIX (1), 11-24. 


\section{INTRODUCCIÓN}

La integración económica es un concepto que ha tomado relevancia en los últimos años, sin que ello signifique que sea nuevo este fenómeno. Según Willhelm Ropke (1942), entre 1700 y 1914, las naciones integraron progresivamente sus economías con la economía mundial; mientras que en el periodo mercantilista se integraron los mercados internos con la intención de disminuir los obstáculos de la economía feudal.

Desde la época de Ropke no se habla sobre integración económica hasta ya entrada la década del 50. Esto es lógico debido a la serie de sucesos bélicos en Europa y en el mundo que socaban los primeros esfuerzos realizados por las naciones del viejo mundo para lograr estrechar relaciones entre ellos. Del final de la Segunda Guerra esta idea sale fortalecida y va a encontrar adeptos que la promovieron, hasta terminar en el comienzo de la actual Unión Europea.

En 1954 el reconocido economista Jan Tinbergen (1968) expone que la integración económica es la combinación de varias economías en una gran unidad territorial, lo cual implica la eliminación de las fronteras económicas de los países, entendiéndose esto último como los obstáculos que impiden la libre movilidad de bienes y de servicios, además de los factores de producción.

Ésta es una definición bastante diciente de integración económica y de lo que implica el término. Pero quien mejor la ha definido y que se acepta hoy en día como la base de la integración económica es Bela Balassa, reconocido economista que definió la integración y sus etapas. A este respecto, el autor expone que la integración económica es un proceso encaminado a abolir las discriminaciones entre las unidades económicas que pertenecen a diferentes Estados (Balassa, 1994, pág. 130). Esto significa e implica que la integración está encaminada a reducir las barreras y crear ambientes de libertad que lleven a una posterior reducción de costos.

Se ve entonces cómo la integración económica es un medio para que los países que se adaptan a esta tendencia pueden fortalecer las relaciones que tienen con otros países, pero además de esto, es un medio que permite acceder más fácilmente a bienes, servicios y factores de producción que en condiciones normales pueden no ser competitivos para el correcto desarrollo de la economía de un Estado.

Claramente van a existir motivaciones que no solo estarán en el campo económico y comercial; hay una estrecha relación con los objetivos políticos y sociales que persigue un gobierno. Todo este conjunto de metas y mezclas de ideología es lo que complejiza el fenómeno de la integración y termina creando una serie de "trade off", entendidos estos como las ganancias $\mathrm{y}$ pérdidas resultantes de la integración económica y comercial.

En la actualidad la integración económica se estudia para determinar los efectos positivos o negativos que ésta puede tener en las economías, especialmente en las que se encuentran en desarrollo; ya que la variedad de acuerdos que se han negociado en los últimos años ha llevado a plantear revisiones sobre el modelo y sobre su implementación en las naciones (Reyes, 2018, pág. 3). 
Esta dinámica se ve sustentada en las diferentes etapas del proceso de integración, ya que este fenómeno implica la cesión de soberanía a medida que se avanza en la reducción de las barreras comerciales y se crean espacios más amplios de intercambio económico. Se han definido cuatro etapas en la integración económica que serán descritas posteriormente.

Es por esta razón que vale la pena hacer una contextualización sobre esta materia, partiendo desde la definición de integración económica y comercial, para así ir acotando el término y la materia hacia Colombia y la relación del país con el entorno internacional, específicamente con la Unión Europea. La intención de este artículo es brindar mayor claridad sobre esta dinámica y sus efectos; centrándose en la relación comercial existente entre los dos socios mediante el análisis de los indicadores de posición comercial, los cuales son usados para analizar la dinámica comercial de dos socios y establecer el grado de dependencia existente.

Lo anterior lleva a pensar como pregunta de investigación ¿Cuál es el grado de dependencia existente en el comercio exterior realizado entre Colombia y la Unión Europea? Para intentar dar respuesta a esta pregunta se planteó la siguiente hipótesis: el acuerdo de asociación entre Colombia y la Unión Europea fue negociado para incrementar el flujo comercial y mejorar el crecimiento económico. Sin embargo, la dinámica comercial muestra que las exportaciones de Colombia tienen una alta concentración en productos primarios y el crecimiento económico no ha sido alto como se esperaba.

El objetivo general bajo el que se enmarcó la investigación es determinar el grado de dependencia en el comercio bilateral entre Colombia y la Unión Europea. Para este análisis se recurrió al uso de datos secundarios obtenidos de entidades oficiales nacionales, así como de organizaciones internacionales. Estos datos fueron usados en los indicadores de posicionamiento comercial manejados por la Comisión Económica para América Latina y el Caribe - CEPAL; lo cual demostró que existe una alta dependencia de Colombia con la Unión Europea, tanto en exportaciones como en importaciones.

El artículo inicia con un marco teórico sobre la integración económica y la importancia de estos procesos. En la siguiente sección se expone la metodología a usar, definiendo los indicadores y su composición; para proceder a utilizar las ecuaciones sobre indicadores de posicionamiento comercial y hacer un análisis de los resultados y de la relación comercial bilateral. El artículo finaliza con las conclusiones, mostrando la necesidad de diversificar las exportaciones colombianas.

\section{MARCO TEÓRICO}

En esta sección se describen las etapas de la integración económica y comercial, para brindar un contexto sobre la situación del comercio internacional y la importancia que estos espacios comerciales tienen para los diferentes países, así como la necesidad de mantener relaciones comerciales fluidas con los diferentes bloques económicos.

\subsection{Etapas de la integración económica.}

Como se mencionó en el apartado anterior existen cuatro etapas de la integración. Sin embargo, aparecen dos que complementan este proceso. Para efectos de una mejor comprensión se explicarán las seis etapas, teniendo en cuenta que a medida que se 
avanza en cada una de estas hay ganancias y pérdidas, dentro de las cuales la más notable es la soberanía; pero los gobiernos están dispuestos a hacerlo ya que esperan recibir mayores beneficios (Maesso Corral, 2011, págs. 120-122).

En las preferencias comerciales los países involucrados se otorgan preferencias arancelarias, buscando con esto garantizar un trato más favorable que el realizado con terceros. Como ejemplo de esto se puede ver las preferencias comerciales que se otorgan los países miembros de la ALADI o las preferencias que se encuentran en el marco de los Sistemas Generalizados de Preferencias.

En el caso de la zona de libre comercio los países establecen la reducción total o paulatina de las barreras arancelarias y no arancelarias para bienes y para servicios (Malagón Herrera, 2013, págs. 2-5). Actualmente se incluyen temas como la propiedad intelectual y las compras públicas. Ejemplos de esta fase se tienen en los llamados Tratados de Libre Comercio, la Asociación Europea de Libre Comercio (EFTA por sus siglas en inglés), el NAFTA, entre otros (Balassa, 1964, pág. 22).

En la etapa de unión aduanera a las preferencias comerciales se les suma el establecimiento de un Arancel Externo Común (AEC) para países que no son miembros del tratado (Petit Primera, 2014, págs. 141-142). En esta etapa hay una cesión mayor de soberanía, afectando la capacidad de decisión sobre la política arancelaria y aduanera del país (Krishna, 2013). Ejemplos de este nivel se ven con la Comunidad Andina (CAN), el Mercado Común del Sur (MERCOSUR, aunque su nombre sea Mercado Común en la práctica aún no ha alcanzado esta fase) o la Unión Aduanera Surafricana (SACU por sus siglas en inglés).

Posteriormente, el mercado común adiciona a los compromisos adquiridos anteriormente la libre movilidad de personas y de capitales; con lo cual se completan las llamadas cuatro libertades fundamentales (Villanueva, 1993, págs. 37-39). Actualmente no hay un grupo de integración que haya llegado a este nivel, salvo la Comunidad Económica Europea previa al Tratado de Maastricht.

Finalmente, la unión económica y monetaria implica la creación de instituciones supranacionales que permitan la coordinación y armonización de las políticas que afectan el sistema económico (Visintin, 2007, págs. 5-8). Esto implica la igualación de las políticas cambiarias, monetarias y fiscales. Actualmente es el nivel más alto de integración alcanzado, y lo ostenta la Unión Europea.

En el caso de la unión política, si bien es una etapa que se sale del aspecto económico ya que involucra la armonización del aspecto político y la creación de una única Constitución Política, vale la pena mencionarlo ya que vendría siendo la etapa posterior y final a las etapas mencionadas anteriormente. Actualmente no hay un proceso de integración que pueda clasificarse en este nivel, si bien hay algunos que colocan a Estados Unidos como ejemplo de este proceso, dadas las características políticas de la potencia mundial (Aragón, 2004, págs. 4-5)

\subsection{El libre comercio en Colombia: entre cambios y una política comercial poca clara}

Durante los últimos años las naciones han realizado un cambio completo en cuanto a su 
relación con sus semejantes y principalmente en cuanto a sus políticas de relacionamiento con el exterior. Este cambio se ha sentido con especial fuerza en los países en vías de desarrollo, muchos de los cuales durante años se manejaron con políticas aisladas respecto a la comunidad internacional pero que buscaban un desarrollo interno que les diera la fortaleza para competir posteriormente en el mercado internacional.

Este fue el caso específico de Colombia, desde la década del 50 la política comercial del país se sustentaba en la práctica proteccionista del modelo de Industrialización por Sustitución de Importaciones propuesto por la Comisión Económica para América y el Caribe (CEPAL). Este modelo se sustentaba en una restricción de las importaciones de bienes que competían con la industria nacional; la cual se acompañaba con una fuerte intervención del gobierno en cuanto a la generación de créditos y subsidios a los productores exportadores (Guarín Grisales \& Franco López, 2008, págs. 58-60).

Si bien el modelo permitió la generación de industria temprana en el país, tal como los plásticos, cauchos y neumáticos; la nación se veía afectada por las continuas variaciones cambiarias sustentadas en un tipo de cambio fijo y a las pocas divisas que generaba la exportación de café. Es por esta razón que a fines del 60 se cambia a un modelo mixto de orientación exportadora con la expedición del Decreto Ley 444 de 1967; el cual buscaba la disminución de la dependencia de las divisas del café y crear un sistema de devaluación que llevara a incentivar la marginal práctica exportadora.

El modelo permitió en principio un aumento de la tasa de crecimiento de la industria, la cual registró incrementos del 7\% que llevaron a que la economía colombiana creciera en promedio al 6\%. En este periodo se vio un crecimiento igual de las exportaciones, principalmente de las consideradas menores que alcanzaron un $40 \%$; sin que por ello se generara un desprendimiento de la tendencia de concentración de exportaciones y canasta mono - exportadora que había caracterizado a Colombia (Garay, 2016, págs. 21-22).

Frente a esta recurrente tendencia en la estructura productiva y exportadora del país cabe hacer algunas anotaciones. La situación del país no se debe a las coyunturas internacionales o a la injerencia de terceros países. Como muchos países que cuentan con altas ventajas naturales, se ha generado una fuerte dependencia sobre las mismas con el correr de los años. De la misma manera se puede ver que el comercio del país se sustenta en la Ley de la Ventaja Comparativa de David Ricardo, así como en la teoría de las proporciones factoriales.

Deardoff (2007) plantea entonces que lo anterior se sustenta en que, de acuerdo con Ricardo, un país exportará los bienes en los cuales tiene una ventaja comparativa, determinada ésta gracias a la dotación de factores, pero más aún por el costo de oportunidad. A diferencia de lo planteado previamente por Smith, quien decía que el comercio iba a estar determinado por el valor absoluto de la producción (ventaja absoluta), Ricardo plantea la posibilidad que el comercio se va a desarrollar en virtud de la eficiencia y mejor uso de los recursos de los que dispone una nación (Maneschi, 2008, págs. 1178-1180).

Ahora bien, se reconoce a Ricardo como el padre del comercio internacional y la ventaja 
comparativa como la base de las transacciones actuales2; pero se complementa con las diferentes teorías que se han dado a lo largo de los años. A este respecto uno de los avances más importantes es la teoría de la dotación factorial o Heckscher - Ohlin, la cual plantea que los países comerciarán dependiendo los factores de producción con que cuenta el país (Jones, 1957, pág. 3). Esto va a significar que de acuerdo con las proporciones factoriales, esto es la proporción de un factor respecto al otro, un país va a ser abundante en uno u otro factor y así mismo producirá y exportará los bienes que usen de manera intensiva el factor abundante y por ende barato.

Si se tiene en cuenta que Colombia cuenta con una extensión de tierra superior a 1.1 millones de kilómetros cuadrados y una población cercana a los 45 millones de personas; la proporción estará dada por la fórmula $\mathrm{T} / \mathrm{L}$ o L/T según sea el caso; donde $\mathrm{T}$ es tierra y L trabajo. En el modelo HO después de hallar la proporción en el país se comparará ésta con la proporción de otro país con el cual se tengas relaciones comerciales (Jones, 1957, pág. 5). Para una serie de países determinados, Colombia resultaba teniendo una proporción de tierra superior a la de las naciones con las que se comparaba, generando con esto la conclusión que Colombia producirá y exportará bienes intensivos en tierra dada su mayor proporción y costo.

Cuevas (1994) plantea que esta teoría ha sido ampliamente criticada en la medida que cierra a los países a determinado patrón de producción. Sin embargo, puede darnos una idea al menos en el sector primario de la economía, sobre cómo podrían estar

2 En los Principios de Economía Política y Tributación Ricardo analiza las transacciones económicas de la época y lleva los postulados liberales de Adam Smith a un nivel mayor, y con los estructuradas las exportaciones y llevar a concluir que las exportaciones colombianas estarán concentradas en productos del sector agrícola, donde se puede decir además que podría poseer una ventaja comparativa en estos productos.

Esto lleva a pensar también en la teoría de la internacionalización y cómo las diferentes aproximaciones que se han hecho sobre ésta pueden ayudar a aprovechar de mejor manera el comercio internacional y los acuerdos negociados. Entender la demanda en el país destino, la posibilidad de personalizar los productos para determinados clientes $\mathrm{o}$ incluso llegar a desarrollar la internacionalización como parte de un proceso específico para el cliente, son aspectos que deben estudiar las empresas para incrementar el aprovechamiento de lo establecido en los diferentes acuerdos comerciales suscritos (Fonseca Cifuentes, 2013, págs. 57-58).

En la siguiente sección se mostrará a través de diferentes indicadores de comercio exterior una serie de interpretaciones y conclusiones para el caso colombiano y su relación con la Unión Europea.

\section{Metodología}

Para el desarrollo de esta investigación se recurrió al uso de datos secundarios obtenidos del Departamento Administrativo Nacional de Estadística - DANE, y de la base de datos TradeMap. Esos datos fueron analizados y reemplazados en las ecuaciones que son parte de los indicadores de comercio exterior. Se usaron dos indicadores que son los que muestran el grado de dependencia frente a un

costos de oportunidad logra garantizar el desarrollo del comercio internacional a través de la creación de ventajas comparativas en las naciones. 
mercado específico y la ventaja que se tiene frente a los competidores externos. Los indicadores usados fueron el Índice Herfindahl - Hirschman y la ventaja comparativa revelada; ya que ambos correlacionan el comercio exterior de los socios comerciales y determinan donde se encuentra concentrado el comercio de estos, además de determinar las ventajas que tiene un socio frente a otro.

La fórmula usada en el IHH expresa las exportaciones de un producto en un periodo específico sobre el total de las exportaciones de ese país; elevado al cuadrado. Para eliminar posibles distorsiones derivadas del número de sectores se suele hacer la normalización del índice.

$$
\begin{array}{r}
I H H=\sum_{i=1}^{n}\left(\frac{x s_{i}^{k}}{x s_{i f}}\right)^{2}, \\
\text { IHHnorm }=\frac{I H H-\frac{1}{n}}{1-\frac{1}{n}} .
\end{array}
$$

El arancel de aduanas o sistema armonizado cuenta con 97 capítulos para Colombia, en esta evaluación se tomaron los diez primeros que es donde se observan las mayores diferencias y variaciones.

Este indicador propuesto por Balassa mide el peso del producto en las exportaciones de bienes del país, sobre el peso que tiene este comercio sobre el del mundo o en este caso puntual el de la Unión Europea. Si el índice es superior a uno significa que hay ventaja comparativa en ese producto, ya que, en relación con el total exportado, exporta más que el mundo a ese mercado.

\section{RESUltados}

\subsection{Indicadores de comercio exterior}

Para determinar los beneficios y perjuicios que tiene la relación comercial bilateral, y más aún lo negociado en el marco de un Acuerdo de Asociación, hay que hacer un análisis objetivo y en la medida de lo posible sustentado con datos estadísticos, los cuales pueden ser usados en indicadores de comercio exterior que permitan determinar el grado de penetración de importaciones o la apertura exportadora de un país.

De acuerdo con diferentes indicadores obtenidos de la CEPAL se puede realizar dicha inferencia, pero no solo por esto se puede determinar el verdadero impacto de los acuerdos comerciales, más aún cuando en el caso del Acuerdo entre Colombia y la Unión Europea solo han pasado cuatro años desde su entrada en vigor y los planes para su aprovechamiento recién se están poniendo en práctica.

Para este análisis entonces se han tenido en cuenta datos de los principales agregados económicos como lo son el Producto Interno Bruto, la población, las exportaciones e importaciones totales y por producto a nivel de capítulo del arancel de aduanas.

Dentro de este estudio vale la pena comenzar entonces por mostrar la evolución del Producto Interno Bruto así como de las exportaciones y las importaciones colombianas durante el periodo 2002 - 2018; si bien el periodo de estudio en este trabajo es menor, vale la pena mostrar la evolución de estos agregados desde un año antes del lanzamiento de las negociaciones comerciales bilaterales; esto con el fin de poder hacer una idea general sobre el contexto en el cual se desarrolla el comercio exterior colombiano. 
Análisis del comercio en Colombia: Unión Europea desde el uso de los indicadores de comercio

exterior

TABLA 1. EXPORTACIONES E IMPORTACIONES COMO PORCENTAJE DEL PIB. EN MILES DE MILLONES COP.

\begin{tabular}{rrrrrr}
\hline & $\begin{array}{r}\text { PIB a precios } \\
\text { corrientes }\end{array}$ & Exportaciones totales & Importaciones totales & Exportaciones/PIB & Importaciones/PIB \\
\hline $\mathbf{2 0 0 2}$ & 245.323 & 36.346 & 44.568 & 0.15 & 0.18 \\
$\mathbf{2 0 0 3}$ & 272.345 & 45.142 & 54.308 & 0.17 & 0.20 \\
$\mathbf{2 0 0 4}$ & 307.762 & 51614 & 58.760 & 0.17 & 0.17 \\
$\mathbf{2 0 0 5}$ & 340.156 & 57.316 & 63.896 & 0.18 & 0.19 \\
$\mathbf{2 0 0 6}$ & 383.898 & 67.710 & 78.839 & 0.17 & 0.21 \\
$\mathbf{2 0 0 7}$ & 431.072 & 71.296 & 85.312 & 0.18 & 0.20 \\
$\mathbf{2 0 0 8}$ & 480.087 & 85.405 & 97.278 & 0.16 & 0.20 \\
$\mathbf{2 0 9}$ & 504.647 & 80.899 & 92.094 & 0.16 & 0.18 \\
$\mathbf{2 0 1 0}$ & 544.924 & 86.839 & 96.805 & 0.19 & 0.18 \\
$\mathbf{2 0 1 1}$ & 619.894 & 116.144 & 123.562 & 0.18 & 0.20 \\
$\mathbf{2 0 1 2}$ & 664.240 & 121.282 & 133.096 & 0.18 & 0.20 \\
$\mathbf{2 0 1 3}$ & 710.257 & 125.114 & 143.620 & 0.14 & 0.20 \\
$\mathbf{2 0 1 4}$ & 762.903 & 109.181 & 127.557 & 0.12 & 0.17 \\
$\mathbf{2 0 1 5}$ & 804.692 & 97.998 & 146.664 & 0.11 & 0.18 \\
$\mathbf{2 0 1 6}$ & 863.782 & 96.614 & 136.678 & 0.12 & 0.16 \\
$\mathbf{2 0 1 7}$ & 920.194 & 111.835 & 135.983 & 0.13 & 0.15 \\
$\mathbf{2 0 1 8}$ & 976.056 & 123.627 & 151.220 & 0.15 \\
\hline
\end{tabular}

Fuente: Cálculos propios basados en información del Departamento Administrativo Nacional de Estadística - DANE

Como se puede ver en la tabla 1 y en el gráfico 1, el comercio exterior colombiano no es alto ni en valores absolutos y mucho menos como proporción del PIB.Esto muestra una debilidad enorme en la estructura productiva y en la inserción en las cadenas globales de valor, ya que la generación de producto no es alta y se puede concluir que la capacidad de compra de importaciones es igualmente baja; además se ve una disminución constante en los montos de comercio exterior realizados por el país.

GRAFICA 1. EXPORTACIONES/PIB E IMPORTACIONES/PIB EN VALORES PORCENTUALES.

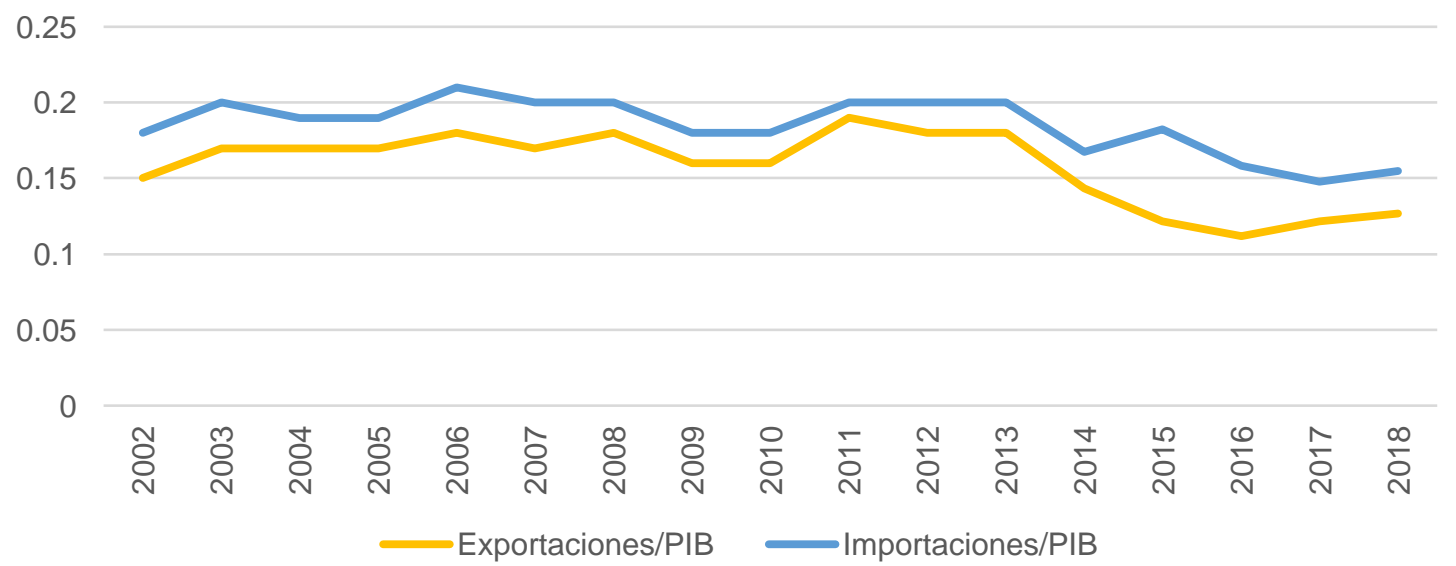

Fuente: Elaboración propia con base en información del Departamento Administrativo Nacional de Estadística - DANE

Ahora bien, si se analiza el comercio exterior per cápita, se puede ver que en promedio los colombianos pasaron de comerciar cerca de 2 millones en 2002 a cerca de 5 millones en 2017. Si bien ha habido un aumento constante del comercio por persona, 
las cifras no son constantes ya que se soportan principalmente en los precios de exportación de commodities y son altamente sensibles a las coyunturas internacionales. Lo anterior muestra igualmente que Colombia es un país importador por naturaleza y dependiente del sector externo.

GRAFICA 2. EXPORTACIONES E IMPORTACIONES POR HABITANTE, EN PESOS COLOMBIANOS.

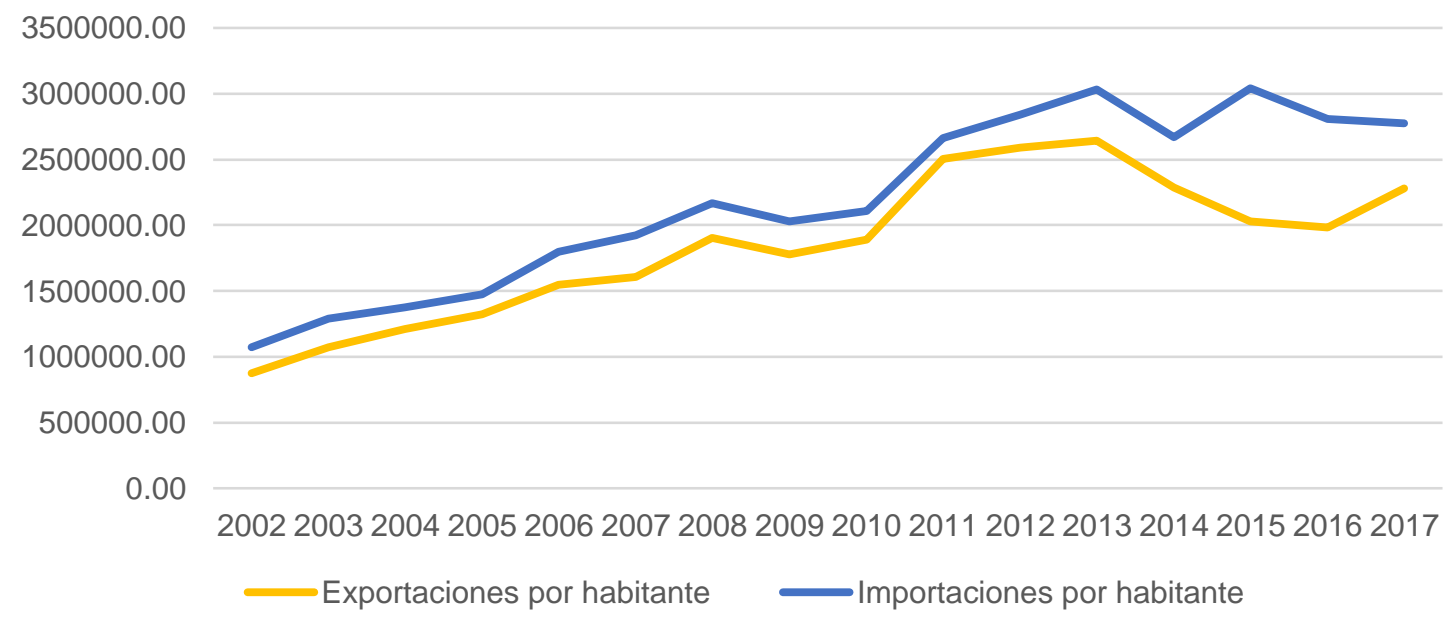

Fuente: Elaboración propia con base en información del Departamento Administrativo Nacional de Estadística - DANE

La gráfica 2 refleja la posición e importancia de Colombia para el comercio internacional, en la cual se puede ver que la participación de las exportaciones e importaciones del país en los totales mundiales es mínima y ni aún la suma de éstas lleva a que el comercio exterior colombiano sea el $1 \%$ del mundial; lo cual permite concluir en concordancia con lo anteriormente expuesto que Colombia está alejado del comercio internacional y es poco competitivo en el desarrollo de este.

GRAFICA 3. PARTICIPACIÓN DE COLOMBIA EN EL COMERCIO MUNDIAL

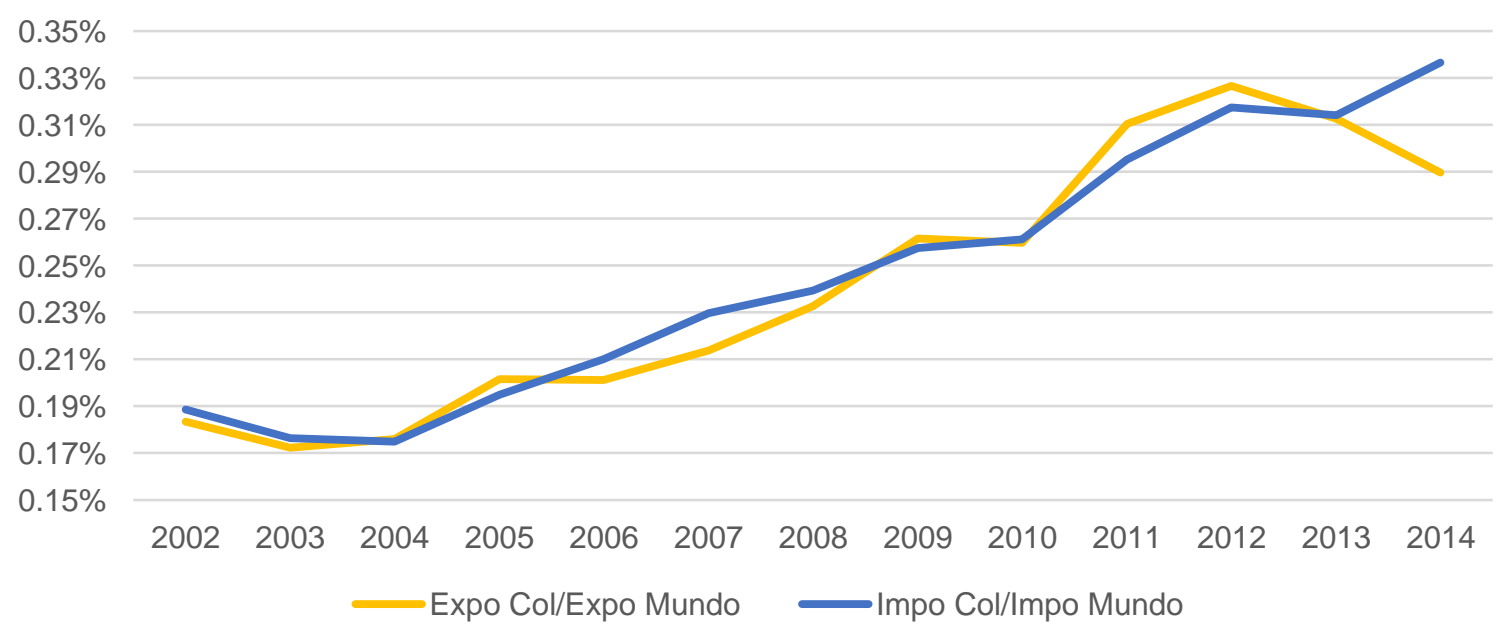

Fuente: Elaboración propia con base en información del Departamento Administrativo Nacional de Estadística - DANE. 


\subsection{Comercio Bilateral Colombia - Unión Europea.}

La Unión Europea ha sido uno de los principales mercados de exportación para los productos colombianos, así como una fuente importante de bienes importados que complementan la estructura productiva nacional. Claramente la Unión Europea (en adelante UE) tiene una importancia enorme para el desarrollo y evolución del comercio exterior colombiano, sin embargo, en los últimos años esta importancia se ha visto afectada por el ascenso de nuevos actores.

Conforme a cifras obtenidas del DANE, las cuales se presentan en la tabla 2 , el segundo mercado para las exportaciones colombianas es la UE, región que durante muchos años ha ocupado esta posición, por encima de mercados naturales como el andino o el latinoamericano. Es importante mencionar que los principales productos que se exportan son productos de la industria mineroenergética, café y banano.

Hay que tener en cuenta que esta dinámica comercial es bastante peligrosa para Colombia, en la medida que está a merced de los cambios en los precios internacionales de los bienes primarios; precios que suelen ser volátiles y que impactan de manera muy fuerte en la dinámica de las exportaciones del país.

TABLA 2. DESTINO DE LAS EXPORTACIONES COLOMBIANAS. CIFRAS EN MILLONES DE USD (FOB).

\begin{tabular}{lrrrrrrr}
\hline Destino & $\mathbf{2 0 1 2}$ & $\mathbf{2 0 1 3}$ & $\mathbf{2 0 1 4}$ & $\mathbf{2 0 1 5}$ & $\mathbf{2 0 1 6}$ & $\mathbf{2 0 1 7}$ & $\mathbf{2 0 1 8}$ \\
\hline Total Exportaciones & 60.125 & 58.826 & 54.857 & 36.018 & 31.768 & 37.881 & 41.831 \\
Estados Unidos & 21.833 & 18.462 & 14.224 & 10.008 & 10.286 & 10.553 & 10.616 \\
Unión Europea & 9.129 & 9.290 & 9.413 & 6.008 & 4.971 & 5.439 & 4.894 \\
Can & 3.618 & 3.392 & 3.213 & 2.768 & 2.399 & 2.759 & 3.160 \\
Mercosur & 4.169 & 4.321 & 3.850 & 2.453 & 1.841 & 2.004 & 2.292 \\
Ecuador & 1.910 & 1.975 & 1.888 & 1.470 & 1.200 & 1.465 & 1.852 \\
México & 835 & 864 & 914 & 914 & 937 & 1.537 & 1.638 \\
Japón & 360 & 388 & 421 & 520 & 428 & 557 & 474 \\
Venezuela & 2.556 & 2.256 & 1.987 & 1.060 & 614 & 319 & 354 \\
\hline
\end{tabular}

Fuente: Departamento Administrativo Nacional de Estadística - DANE

En el caso de las importaciones, la tabla 3 muestra que, si bien la Unión Europea es uno de los socios históricamente más importantes para Colombia, ha venido perdiendo terreno frente al ascenso de China como proveedor de diferentes tipos de bienes manufacturados. Sin embargo, aún mantiene una gran importancia debido a que muchos bienes industriales y de capital siguen siendo demandados.

Esta relación comercial sustentada en la información oficial muestra que los dos socios son complementarios y que además cuentan con una rica historia de cooperación $y$ relaciones comerciales. Si bien para Colombia esta relación es de suma importancia, para la Unión Europea es prácticamente residual; pero el análisis debe ir más allá respecto a nuestra concentración en este mercado y los productos que se envían. Para profundizar un poco más en este análisis se propone el uso de dos indicadores de comercio exterior, tanto para analizar la diversificación de comercio como la ventaja comercial que tiene el país.

Ahora bien, al usar los indicadores de comercio internacional se pueden encontrar hallazgos sobre el proceso que ha venido adelantando Colombia en los últimos años y específicamente con la Unión Europea. 
TABLA 3. ORIGEN DE LAS IMPORTACIONES COLOMBIANAS. CIFRAS EN MILLONES DE USD (CIF).

\begin{tabular}{lrrrrrrr}
\hline Origen & $\mathbf{2 0 1 2}$ & $\mathbf{2 0 1 3}$ & $\mathbf{2 0 1 4}$ & $\mathbf{2 0 1 5}$ & $\mathbf{2 0 1 6}$ & $\mathbf{2 0 1 7}$ & $\mathbf{2 0 1 8 ^ { * }}$ \\
\hline Total Importaciones & 59.048 & 59.381 & 64.029 & 54.058 & 44.889 & 46.076 & 51.231 \\
Estados Unidos & 14.178 & 16.337 & 18.193 & 15.512 & 11.878 & 12.014 & 12.986 \\
China & 9.822 & 10.363 & 11.790 & 10.032 & 8.631 & 8.754 & 10.545 \\
Unión Europea & 7.359 & 7.949 & 8.761 & 8.278 & 6.301 & 6.855 & 7.583 \\
México & 6.453 & 5.496 & 5.273 & 3.853 & 3.411 & 3.437 & 3.947 \\
Mercosur & 5.955 & 4.904 & 4.050 & 2.985 & 2.950 & 3.133 & 3.779 \\
Can & 2.301 & 2.300 & 2.675 & 2.153 & 2.021 & 1.731 & 1.893 \\
Japón & 1.677 & 1.479 & 1.525 & 1.227 & 1.116 & 1.231 & 1.289 \\
Venezuela & 533 & 431 & 440 & 292 & 190 & 220 & 137 \\
\hline
\end{tabular}

Fuente: Departamento Administrativo Nacional de Estadística - DANE

A continuación, se muestra en la tabla 4 el grado de diversificación exportadora construido con el Índice Herfindahl Hirschman, el cual determina cuan concentrado se encuentra un producto en un mercado; cuando el resultado arroja un número por encima de 0,18 se considera concentrado y por debajo de 0,18 diversificado.

Para las exportaciones de Colombia a la Unión Europea posteriores a la entrada en vigor del acuerdo se ve que hay una alta diversificación en la casi totalidad de los capítulos del arancel de aduanas, salvo en el capítulo de combustibles minerales, donde el indicador se sitúa en un promedio de 0,3. Esto significa que para el sector de minería y combustibles minerales uno de sus mercados naturales e importantes es la Unión Europea, lo cual convierte a este sector en dependiente de este continente; mientras que para los demás sectores o productos hay una mayor diversificación.

TABLA 4. ÍNDICE HERFINDAHL - HIRSCHMAN PARA COLOMBIA Y LA UNIÓN EUROPEA.

\begin{tabular}{|c|c|c|c|}
\hline Capítulo del arancel & 2015 & 2016 & 2017 \\
\hline $\begin{array}{l}27 \text { - Combustibles minerales y productos de su } \\
\text { destilación }\end{array}$ & 0.35682438 & 0.26488194 & 0.28074736 \\
\hline 08 - Frutas y frutos comestibles & 0.00317493 & 0.01672745 & 0.01434564 \\
\hline 09 - Café, té, yerba mate y especias & 0.00670483 & 0.01257648 & 0.00611270 \\
\hline 15 - Grasas y aceites animales o vegetales & -0.00918112 & -0.00867823 & -0.00757420 \\
\hline 06 - Plantas vivas y productos de la floricultura & -0.00996292 & -0.00978756 & -0.00986550 \\
\hline 21 - Preparaciones alimenticias diversas & -0.01033686 & -0.01028734 & -0.01030919 \\
\hline 17 - Azúcares y artículos de confitería & -0.01036075 & -0.01030893 & -0.01031514 \\
\hline 72 - Fundición, hierro y acero & -0.01029463 & -0.01038276 & -0.01032748 \\
\hline 39 - Plástico y sus manufacturas & -0.01035153 & -0.01034451 & -0.01035826 \\
\hline 26 - Minerales metalíferos, escorias y cenizas & -0.01039804 & -0.01038527 & -0.01036202 \\
\hline
\end{tabular}

Fuente: Elaboración propia con base en información de Trade Map

En el caso de la ventaja comparativa revelada, en la tabla 5 se puede ver este indicador que muestra el grado de especialización exportadora de un país; definido como el cociente entre la participación de un producto en las exportaciones de un país y la participación de ese producto en las exportaciones mundiales. Para este caso puntual se han usado los capítulos del arancel de aduanas y se han tomado las exportaciones del mundo hacia la Unión Europea, esto con el fin de analizar el 
grado de importancia de este mercado para Colombia.

Los resultados muestran una tendencia similar a la del índice anterior, en la cual los productos primarios tienen una alta participación en el comercio bilateral, teniendo una clara ventaja comparativa como es el caso del café, las frutas (banano especialmente), flores, grasas y combustibles. También se han querido mostrar otros productos que, aunque no tienen una ventaja tan clara, están cerca de tenerla dada la demanda de la Unión Europea por este tipo de productos.

TABLA 5. VENTAJA COMPARATIVA REVELADA COLOMBIA - UNIÓN EUROPEA.

\begin{tabular}{lrrr}
\hline Capítulo del Arancel & $\mathbf{2 0 1 5}$ & $\mathbf{2 0 1 6}$ & $\mathbf{2 0 1 7}$ \\
\hline 09 - Café, té, yerba mate y especias & 33.3339922 & 38.7940608 & 33.8768440 \\
08 - Frutas y frutos comestibles & 12.7923090 & 17.4689284 & 17.1600464 \\
06 - Plantas vivas y productos de la floricultura & 9.4733621 & 10.8097165 & 10.7023816 \\
15 - Grasas y aceites animales o vegetales & 6.5765077 & 7.5656979 & 9.4296672 \\
27 - Combustibles minerales y productos de su destilación & 5.5664608 & 6.0647382 & 5.3838418 \\
17 - Azúcares y artículos de confitería & 3.2945870 & 4.3953266 & 4.4334977 \\
41 - Pieles y cueros & 3.9526344 & 3.1971933 & 2.4973286 \\
21 - Preparaciones alimenticias diversas & 1.9810084 & 2.4031978 & 2.2706342 \\
26 - Minerales metalíferos, escorias y cenizas & 0.7739658 & 1.1503432 & 1.2523314 \\
35 - Materias albuminoideas; productos a base de almidón & 0.5901014 & 0.3382367 & 1.1243654 \\
24 - Tabaco y sucedáneos del tabaco elaborados & 0.8237688 & 0.6302536 & 0.9994356 \\
18 - Cacao y sus preparaciones & 1.0225420 & 1.2230731 & 0.8806423 \\
03 - Pescados y crustáceos, moluscos y demás invertebrados & 0.4100038 & 0.6388783 & 0.7673566 \\
53 - Las demás fibras textiles vegetales & 0.0617431 & 3.0130662 & 0.7350074 \\
43 - Peletería y confecciones de peletería & 0.5112775 & 0.7619495 & 0.5689648 \\
20 - Preparaciones de hortalizas, de frutas u otros frutos & 0.4250856 & 0.4719044 & 0.4930557 \\
74 - Cobre y sus manufacturas & 0.4211340 & 0.4209207 & 0.4669258 \\
72 - Fundición, hierro y acero & 0.4612764 & 0.2598604 & 0.3666346 \\
14 - Materias transables y demás productos de origen vegetal & 0.7739658 & 1.1503432 & 1.2523314 \\
96 - Manufacturas diversas & 0.5901014 & 0.3382367 & 1.1243654 \\
\hline
\end{tabular}

Fuente: Elaboración propia con base en información de Trade Map

\section{CONCLUSIONES}

A lo largo de este artículo se ha realizado una revisión de algunos indicadores de posicionamiento comercial entre Colombia y la Unión Europea, en la cual destaca que, si bien la Unión Europea es un socio comercial importante para Colombia, la participación y el peso que este grupo de integración comercial tiene, aún es bajo, y la presencia de los productos colombianos en el mercado europeo es bastante débil, salvo el caso de los productos primarios o con bajo valor agregado, los cuales tienen una clara ventaja frente a sus similares del continente europeo.Si bien en términos nominales el comercio puede ser importante y más para un país pequeño como Colombia, al usar los indicadores de posición comercial se evidencia que hay una alta concentración de productos primarios en el mercado europeo, cuestión predecible dado nuestro intercambio histórico y nuestra relación permeada por las asimetrías económicas y comerciales. Existe una gran asimetría entre el grupo europeo y Colombia, con el paso de los años el país ha gozado de sistemas de preferencias y un trato más favorable que el dado a otros mercados, pero esto no se ha reflejado en una mejora del aparato productivo del país y mucho menos en una diversificación de la oferta exportable. 
Se puede ver igualmente que no solo el grado de concentración de productos es alarmante, en la medida que se depende casi que exclusivamente de los bienes primarios que se exportan al mercado europeo y que como también se mencionó, tienen un precio altamente volátil como se ha visto en los últimos años.

De la misma manera se puede ver que el país no cuenta con una ventaja clara y redituable frente a las demandas del mercado, son bienes que se producen y que cuentan con un poco o escaso valor agregado y que gracias a esto no revisten mayor importancia para las demandas de un mercado exigente y de alta calidad como el europeo. Dentro del grupo de productos que tienen una clara ventaja en el desarrollo del comercio bilateral se puede apreciar que la gran mayoría son bienes primarios con poco o bajo valor agregado, aunque algunos de estos bienes tienen un proceso mayor como el caso del cacao y sus manufacturas (chocolates) o grasas y aceites; donde claramente hay una actividad industrial mayor $y$ que ha logrado incrementar su componente de valor agregado, diferenciación y competitividad.

Esto también lleva a pensar que de alguna manera el producto colombiano está posicionado en este mercado y ha logrado cierto reconocimiento; la competencia es alta ya que no solo lo procedente de Asia se vende en este mercado, sino la producción de países europeos, como es el caso de la marroquinería, que tiene su principal proveedor en Italia o de las diferentes preparaciones alimenticias, que pueden tener diferentes focos de producción; situaciones que no aseguran que las ventajas obtenidas hasta este momento se puedan mantener en el futuro.
Vale la pena mencionar que, si bien las condiciones políticas se han dado con la firma del acuerdo de asociación con la Unión Europea, a partir de la entrada en vigor de este acuerdo, las exportaciones colombianas se han reducido y las importaciones han aumentado; no es culpa del acuerdo en la medida que las condiciones son de doble vía, hay un problema estructural en la producción colombiana, que limita seriamente las posibilidades de salir y triunfar en mercados internacionales.

Tal como se ha mencionado en diferentes escenarios, el empresariado debe buscar la manera de mejorar su proceso productivo y crear productos con valor agregado que se posicionen en el mercado europeo. Igualmente, el gobierno debe continuar con las acciones de modernización en la infraestructura logística y de mejora del aparato legal, para con esto crear condiciones competitivas que no generen obstáculos al momento de exportar. Adicional a esto se debe invertir y profundizar en el uso de tecnología en la producción y en el servicio al cliente; la diferenciación no solo se da en el producto sino en la inclusión de nuevas tecnologías que permitan incrementar el valor agregado en cada una de las partes de la cadena de valor.

Esto no es proceso que se pueda lograr en poco tiempo, pero la acción mancomunada entre los diferentes actores de la economía puede permitir que se genere el cambio disruptivo que permita mejorar la posición de los productos colombianos en vez del retroceso que se está viendo cada año. Esto lleva a pensar en nuevas estrategias para las empresas colombianas de cara a afrontar los mercados internacionales, pensando no solamente en la simple atención de un mercado sino en la real satisfacción de los 
clientes, en desagregar la producción o incluso en la producción basada en proyectos; estrategias que podrían ser evaluadas en el futuro contra la dinámica de las exportaciones colombianas.

\section{REFERENCIAS}

Aragón, E. (2004). Estados de la Integración Económica. Obtenido

de http://www.oirsa.org/aplicaciones/subidoarchivos/Bibliot ecaVirtual/Estadosdelaintegracioneconomica.pdf

Balassa, B. (1964). Commodity Movements: Productions Aspects. En B. Balassa, The Theory of Economic Integration (págs. 21-56). New York: Routledge Revivals.

Balassa, B. (1994). The Theory of Economic Integration: An introduction. En B. Balassa, The European Union: Readings on the Theory and Practice of European Integration (págs. 125-137). Londons: McMillan Education.

Cuevas, H. (1994). Una explicación alternativa de la paradoja de Leontieff. Cuadernos de Economía, 157-163.

Deardoff, A. (2007). The Ricardian Model. New Jersey: Princeton Encyclopedia of the World Economy.

Fonseca Cifuentes, G. (2013). Teoría de la internacionalización y de los negocios internacionales: una visión desde la organización. Inquietud Empresarial, XIII(1), 49-62.

Garay, L. J. (10 de 10 de 2016). De la sustitución de importaciones a la apertura, 1967-1996. En L. J. Garay, Colombia: Estructura Industrial e Internacionalización 1967-1996 (págs. 19-64). Bogotá: Departamento Nacional de Planeación. Obtenido de Plan Vallejo: http://www.banrepcultural.org/blaavirtual/economia/ind ustrilatina/162.htm

Guarín Grisales, A., y Franco López, D. (2008). La sustitución de importaciones como medio para un desarrollo sostenible. Revista Universidad EAFIT, 56-67.

Jones, R. (1957). Factor proportions and the Heckscher-Ohlin Theorem. The review of economic studies, 21 No 1, 1-10. Obtenido de www.jstor.org/stable/2296232

Krishna, P. (2013). Preferential Trade Agreements: Theory and Evidence. In D. Bernhofen, R. Falvey, D. Greenaway, \& U. Kreickemeier, Palgrave Handbook of International Trade (pp. 349-366). Londes: Palgrave Macmillan. doi:doi.org/10.1007/978-0-230-30531-1_12

Maesso Corral, M. (2011). La Integración Económica. Tendencias y nuevos desarrollos de la teoría económica, $858,119-132$
Malagón Herrera, D. (2013). Colombia y los procesos de integración económica. Universidad Militar Nueva Granada, 2-25.

Maneschi, A. (2008). How would David Ricardo have tought the principle of comparative advantage? Southern Economic Journal, 74(4), 1167-1176. doi:10.2307/20112020

Petit Primera, J. G. (2014). La teoria económica de la integración y sus principios fundamentales. Revista venezolana de análisis de coyuntura, 137-162.

Picanco, E., Ramos Barreto, C., y Barreto Pinto, C. (2015). Internationalization of Small Business: An Investigation about Brazilian Business in Orlando City. Journal of Management Policy and Practice, 16(3), 78-94.

Pugell, T. (2010). La teoría básica: oferta y demanda. En T. Pugell, Economía Internacional (págs. 18-34). Madrid: Mc Graw Hill.

Reyes, G. (2018). Fundamentos de Integración en América Latina. (págs. 1-9). Bogotá: Universidad del Rosario.

Ropke, W. (1942). En W. Ropke, International Economic Desintegration. Edimburgo: Hodge.

Tinbergen, J. (1968). En J. Tinbergen, Integración Económica Internacional. Barcelona: Sagitario.

Tugores, J. (2007). Integración Económica. En J. Tugores, Economía Internacional (págs. 109-144). Madrid: Mc Graw Hill.

Villanueva, J. (1993). Los esquemas análiticos de integración. Boletín de lecturas sociales y económicas, 33-56.

Visintin, S. (2007). La integración económica y el crecimiento, teoría y evidencia empírica en la UE. Instituto de Análisis Económico y Social, 3-26. 